\title{
Applying the disciplinary relation matrix to multidisciplinary design optimization modeling and solving
}

\author{
Hua Su*, Liangxian Gu, and Chunlin Gong \\ Shaanxi Aerospace Flight Vehicle Design Key Laboratory, Northwestern Polytechnical University, Xi'an 710072, China
}

\begin{abstract}
A new efficient coupling relationship description method has been developed to provide an automated and visualized way to multidisciplinary design optimization (MDO) modeling and solving. The disciplinary relation matrix (DRM) is proposed to describe the coupling relationship according to disciplinary input/output variables, and the MDO definition has been reformulated to adopt the new interfaces. Based on these, a universal MDO solving procedure is proposed to establish an automated and efficient way for MDO modeling and solving. Through a simple and convenient initial configuration, MDO problems can be solved using any of available MDO architectures with no further effort. Several examples are used to verify the proposed MDO modeling and solving process. Result shows that the DRM method has the ability to simplify and automate the MDO procedure, and the related MDO framework can evaluate the MDO problem automatically and efficiently.
\end{abstract}

Keywords: multidisciplinary design optimization (MDO), problem definition, solution architecture, solving automation.

DOI: $10.21629 /$ JSEE.2017.04.10

\section{Introduction}

Multidisciplinary design optimization (MDO) has the ability for efficiently organizing the design process and obtaining the consistent optimal solution. Many research studies have been carried out on this topic and made great progress [1,2]. Despite these advances, the design of complex engineering systems remains a challenge $[3,4]$.

Especially in the MDO solving procedure, although many successful cases have been done in recent years, solving a real-world engineering system is still a challenging work. We suffer from the following problems.

(i) There are many different MDO models with various defined variables and MDO architectures, an abundant

Manuscript received December 22, 2015.

*Corresponding author.

This work was supported by the National Natural Science Foundation of China (51505385), Shanghai Aerospace Science and Technology Innovation Foundation (SAST2015010), and the Defense Basic Research Program (JCKY2016204B102). of work are needed to transform the engineering problem to the corresponding MDO solving formulation by taking manual steps. (ii) An MDO problem defines as a function of MDO variables, but has no direct relevance with the discipline model. Adding/removing disciplines and connecting/disconnecting interdisciplinary couples require extensive job, even some subversive rework. (iii) The MDO definition derived from coupling relationship is a complicated mathematical model. Modification and variation of this MDO model need designers with specialized knowledge. (iv) For a special MDO problem, it is necessary to make a comparison and analysis of these MDO architectures to find out a suitable one with efficiency and precision, so how to switch among these solution architectures efficiently and automatedly is worth studying. The application and promotion of MDO have been hampered by these fussy MDO problem definition and the complex solving procedure, until now many MDO researches are confined to the multidisciplinary integrated simulation with no couples and the solution with single MDO architectures [3]. Therefore, the purpose of our study is to seek a more flexible way to describe the MDO problem, reconstruct MDO architecture and automatic solution.

The structure of this paper is as follows. Section 2 introduces a new MDO coupling information description based on disciplinary relation matrix (DRM). Section 3 proposes the MDO mathematical definition with disciplinary input/output variables and establishes the MDO solving procedure. Section 4 gives a brief description for the software design, which is detailed in [5]. Section 5 tests three MDO benchmarks and gives a detailed discussion on this new MDO environment. Finally, Section 6 gives a conclusion and prospect of this research.

\section{MDO modeling by DRM}

\subsection{DRM description}

An essence of MDO is the interdisciplinary coupling re- 
lationship. The reasonability and conciseness of the MDO definition determines the complexity of the MDO solution procedure. The most common way of doing this is to set up the design structure matrix (DSM) and the functional dependence table (FDT). They are both based on matrix description.

DSM is a two-dimensional square with disciplines laying in the diagonal; other elements of the square represent the link of related disciplines. Steward first proposed the DSM method to describe the dataflow of a coupling system [6]. Browning reviewed four DSM applications useful to product developers, project planners, project managers, system engineers, and organizational designers, also a detail summary had been made for the coupling analysis of complex systems [7]. Andrew made a useful comparison of the MDO coupling description methods, and presented a new diagram for visualizing MDO, the extended design structure matrix (XDSM), which showed data dependency and process flow on a single diagram simultaneously based on the DSM [8]. Other researches of DSM also can be found in [9-12]. Though DSM offers a clear way to express the coupling relationship between disciplines, which provides an explicit dataflow relation and the scales of iteration, the detail relationship between the disciplinary input variables and output variables cannot be obtained from the DSM.

FDT [13], also named dependency matrix [14], is a dependency table between design functions and design variables. It is represented by a Boolean matrix, rows are labeled with relation/function names and columns are labeled with variable names. The entry in the $i$ th row and $j$ th column is "true" if the $i$ th function depends on the $j$ th variable; otherwise, it is "false". Kusia et al. first used it for describing the relation between functions and variables, and constructed the disciplines or mission block matrix by linking relation [15]. Numerous studies are carried out to the MDO problem decomposition and reconstruction based on the FDT $[13,16,17]$, for reducing the degree of coupling and the scale of the system. However, there is no universality on the new discipline models reconstructing from the FDT, especially for black-box discipline models come from commercial software and custom codes due to the inseparability between design variables and functions.

DSM and FDT methods cannot describe the MDO coupling characteristics comprehensively, and it is difficult to constitute an automatic MDO solving environment based on those methods. For this reason, our work presented in this paper focuses on a new MDO problem description method based on the DRM, which will provide an efficient and automatic way for MDO modeling, solving and evaluating. This allows the designers to care for the MDO problem itself and lets the developers to focus on new algorithms or methods they are developing and testing, rather than on implementation details.

DSM gives no information on the coupling relationship of the disciplinary input and output variables, and the FDT structure excludes the original discipline information, both cannot describe the coupling relationship of the MDO system comprehensively. In addition, the designer needs a deep insight into the MDO problem for a proper selection of the MDO architectures and has to select the MDO variables manually, which is easy to cause misunderstanding. Therefore, we present an efficient and convenient way for the MDO coupling relationship description based on the disciplinary input and output variables. It is a format based on matrix both containing the disciplinary information and the coupling relationship of the disciplinary input and output variables, so we call it DRM. A three-discipline MDO problem is utilized to illustrate the DRM.

We suppose the disciplinary input and output variables of the three-discipline MDO problem are as follows:

$$
\begin{gathered}
{\left[y_{1}, y_{2}, y_{3}, y_{4}\right]=A\left(x_{1}, x_{2}, x_{3}\right)} \\
{\left[y_{5}, y_{6}, y_{7}\right]=B\left(x_{1}, x_{3}, x_{4}, x_{5}, x_{6}\right)} \\
{\left[y_{8}, y_{9}\right]=C\left(x_{1}, x_{4}, x_{7}\right)}
\end{gathered}
$$

$A, B, C$ are the disciplines with input variables and out-

\begin{tabular}{|c|c|c|c|c|c|c|c|c|c|c|c|c|}
\hline \multirow{2}{*}{ DISP } & \multirow{2}{*}{ Name } & \multicolumn{3}{|c|}{$\boldsymbol{X}_{1}$} & \multicolumn{5}{|c|}{$\boldsymbol{X}_{2}$} & \multicolumn{3}{|c|}{$\boldsymbol{X}_{3}$} \\
\hline & & $x_{1}$ & $x_{2}$ & $x_{3}$ & $x_{1}$ & $x_{3}$ & $x_{4}$ & $x_{5}$ & $x_{6}$ & $x_{1}$ & $x_{4}$ & $x_{7}$ \\
\hline \multirow{4}{*}{$\boldsymbol{Y}_{1}$} & $y_{1}$ & & & & & & & & 0 & & & \\
\hline & $y_{2}$ & & & & & & & & & & & \\
\hline & $y_{3}$ & & & & & & & & & & & \\
\hline & $y_{4}$ & & & $a a$ & & & & & $b a$ & & & $c a$ \\
\hline \multirow{3}{*}{$\boldsymbol{Y}_{2}$} & $y_{5}$ & & & & & & & & & & & \\
\hline & $y_{6}$ & & & & & & & & & & & 0 \\
\hline & $y_{7}$ & & & $a b$ & & & & & $b \boldsymbol{b}$ & & & $c b$ \\
\hline \multirow{2}{*}{$\boldsymbol{Y}_{3}$} & $y_{8}$ & & & 0 & & 0 & & & & & & \\
\hline & $y_{9}$ & & & $a c$ & & & & & $b c$ & & & $c c$ \\
\hline \multicolumn{2}{|c|}{ ShareVar } & 1 & & & 1 & & 2 & & & 1 & 2 & \\
\hline
\end{tabular}
put variables. The DRM of the three-discipline MDO problem is shown in Fig. 1.

Fig. 1 DRM of the three-discipline MDO problem

$\boldsymbol{X}_{1}, \boldsymbol{X}_{2}$ and $\boldsymbol{X}_{3}$ are the vectors of disciplinary input variables, $\boldsymbol{Y}_{1}, \boldsymbol{Y}_{2}$ and $\boldsymbol{Y}_{3}$ are the vectors of disciplinary output variables. The blocks expect main diagonal, such as $\boldsymbol{b} \boldsymbol{a}, \boldsymbol{c a}, \boldsymbol{a b}, \boldsymbol{c b}, \boldsymbol{a c}$ and $\boldsymbol{b c}$, are the regions of interdisciplinary coupling. For instance, $\boldsymbol{b} \boldsymbol{a}$ is the relation matrix consisting of the relationship between the discipline $A$ and discipline $B$, which means the output variables of the discipline $A$ connect to the input variables of the discipline $B$. The circle mark in $\boldsymbol{b} \boldsymbol{a}$ represents the coupling relation between the output variable $y_{1}$ of the discipline $A$ and the 
input variable $x_{6}$ of the discipline $B$. Blank means no coupling relationship. The definition of others is similar too. The last row of the DRM is used to define the share design variables. The input variables in the last row containing the elements with the same value are identified as the share design variable, such as $x_{1}$ of the discipline $A$ and $x_{1}$ of the discipline $B$, these are marked as "ShareVar 1". The blocks $\boldsymbol{a} \boldsymbol{a}, \boldsymbol{b} \boldsymbol{b}$ and $\boldsymbol{c} \boldsymbol{c}$ are the region of discipline-self relationship, which can be used as the disciplinary Jacobi matrix [18].

The DRM is the fusion and extension of the DSM and FDT. It includes the coupling relationship of the design variables and design responses like FDT, and contains the interdisciplinary information like DSM. The whole dataflow of the MDO system and the coupling relationship of disciplinary input and output variables are contained in DRM, which will give full coupling information for MDO modeling in the next section. The clarity and standard formation are also useful for the MDO automatic solving procedure described in Section 4 later.

\subsection{MDO problem}

The most common MDO problem is described as follows:

$$
\begin{gathered}
\min f(\boldsymbol{x}, \boldsymbol{y}(\boldsymbol{x}, \boldsymbol{y})) \\
\text { w.r.t. } \boldsymbol{x}, \boldsymbol{y} \\
\text { s.t. } h(\boldsymbol{x}, \boldsymbol{y}(\boldsymbol{x}, \boldsymbol{y}))=0 \\
g(\boldsymbol{x}, \boldsymbol{y}(\boldsymbol{x}, \boldsymbol{y})) \leqslant 0
\end{gathered}
$$

$\boldsymbol{x}=\left[\boldsymbol{x}_{0}^{\mathrm{T}}, \boldsymbol{x}_{1}^{\mathrm{T}}, \ldots, \boldsymbol{x}_{N}^{\mathrm{T}}\right]^{\mathrm{T}}$ is the design variables groups including share design variables $\boldsymbol{x}_{0}$ and discipline design variables $\boldsymbol{x}_{i} \cdot \boldsymbol{y}=\left[\boldsymbol{y}_{1}^{\mathrm{T}}, \ldots, \boldsymbol{y}_{N}^{\mathrm{T}}\right]^{\mathrm{T}}$ is the coupling variables. $h$ and $g$ are the equality constraints and nonequality constraints of the MDO problem. The above definition provides a direct recognition to the MDO architecture, but has no direct relevance with the discipline model. For designers, sometimes it is difficult to convert a complex MDO problem into (2), some modification must be done to simplify the MDO modeling process.

The disciplinary input and output variables are the most intuitive interfaces of the MDO problem, and from Section 2 we know, DRM is based on the disciplinary input and output variables and contains full coupling relationship of the MDO problem. This information can be utilized to create a standard process for MDO modeling and formulate the MDO architectures. The MDO problem based on disciplinary input and output variables is defined as in the following,

$$
\begin{gathered}
\min F(\boldsymbol{X}, \boldsymbol{Y}) \\
\text { w.r.t. } \boldsymbol{X}, \boldsymbol{Y} \\
\text { s.t. } H(\boldsymbol{X}, \boldsymbol{Y})=0 \\
G(\boldsymbol{X}, \boldsymbol{Y}) \leqslant 0 \\
\boldsymbol{Y}_{i}=\operatorname{Disp}_{i}\left(\boldsymbol{X}_{i}\right), \quad i=1,2, \ldots, N
\end{gathered}
$$

where $\operatorname{Disp}_{i}$ is the $i$ th discipline model, $\boldsymbol{X}_{i}$ is the vector containing all the input variables of discipline $i, \boldsymbol{X}=$ $\left[\boldsymbol{X}_{0}^{\mathrm{T}}, \boldsymbol{X}_{1}^{\mathrm{T}}, \ldots, \boldsymbol{X}_{N}^{\mathrm{T}}\right]^{\mathrm{T}}$ is the input vector of all disciplines. $\boldsymbol{Y}_{i}$ is the vector containing all the output variables of the discipline $i, \boldsymbol{Y}=\left[\boldsymbol{Y}_{0}^{\mathrm{T}}, \boldsymbol{Y}_{1}^{\mathrm{T}}, \ldots, \boldsymbol{Y}_{N}^{\mathrm{T}}\right]^{\mathrm{T}}$ is the output vector of all disciplines. $F$ is the objective function. $G$ is the inequality constraint and $H$ is the equality constraint. The interactive relationship of the DRM and the MDO architecture is indicated in Fig. 2.

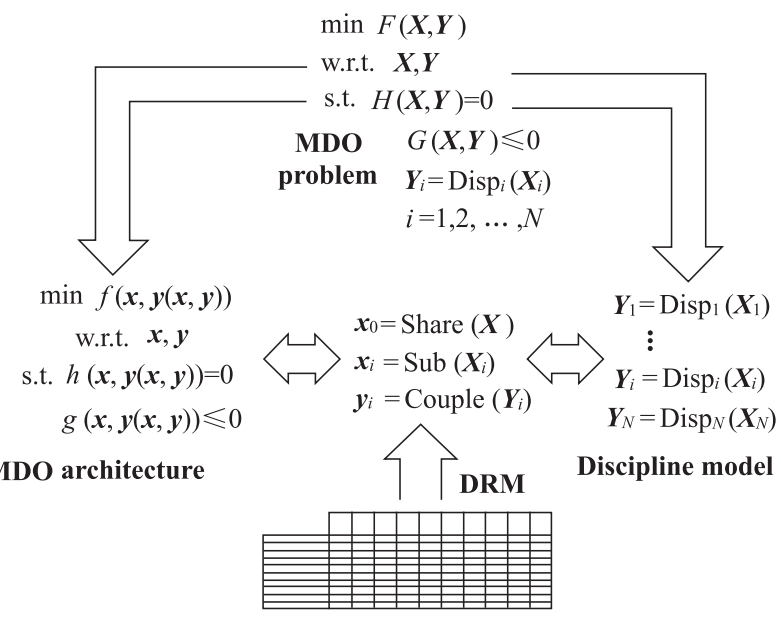

Fig. 2 Interactive relationship of DRM and MDO architecture

The lowercase letters represent the MDO variables, the capital letters represent the disciplinary input/output variables in Fig. 2. The conversion relation between MDO variables and disciplinary input/output variables is listed in Table 1.

Table 1 Conversion between MDO variables and disciplinary input/output variables

\begin{tabular}{cll}
\hline MDO variables & \multicolumn{1}{c}{ Definition } & \multicolumn{1}{c}{ Relation conversion principle } \\
\hline $\begin{array}{c}\text { Discipline } \\
\text { design variable }\end{array}$ & $\begin{array}{l}\text { The one applies only to a certain discipline } \\
\text { as input variables. }\end{array}$ & $\begin{array}{l}\text { The } j \text { th column of the region of interdisciplinary cou- } \\
\text { pling is all blank, e.g. } x_{2}, x_{5} \text { in Fig. 3. }\end{array}$ \\
\hline $\begin{array}{c}\text { Share design } \\
\text { variable }\end{array}$ & $\begin{array}{l}\text { The one is shared by more than one disci- } \\
\text { pline and affects the whole MDO system as } \\
\text { input variables. }\end{array}$ & $\begin{array}{l}\text { There are multiple disciplinary input variables of the } \\
\text { same definition in the last row "ShareVar", e.g. } x_{1}, x_{4} \\
\text { in Fig. 3. }\end{array}$ \\
\hline $\begin{array}{c}\text { Coupling } \\
\text { variable }\end{array}$ & $\begin{array}{l}\text { The one outputs from the discipline analysis } \\
\text { as the input variables of other disciplines. }\end{array}$ & $\begin{array}{l}\text { The } i \text { th row of the region of interdisciplinary coupling } \\
\text { has at least one circle mark, e.g. } y_{1}, y_{6}, y_{8} \text { in Fig. 3. }\end{array}$ \\
\hline
\end{tabular}


$x_{i}=\operatorname{Sub}\left(\boldsymbol{X}_{i}\right), x_{0}=\operatorname{Share}(\boldsymbol{X})$ and $y_{i}=\operatorname{Couple}\left(\boldsymbol{Y}_{i}\right)$ are the conversion functions between discipline design variable, share design variable, coupling variable and the input/output variables of the discipline $i$. The copies of the coupling variable are also called target variable when these copies have been made to allow discipline analyses to run independently and in parallel. The coupling variable is unique, but may have multiple target variables. The formula of MDO architectures also needs some modification to adapt the DRM interface, which will be introduced in the next section.

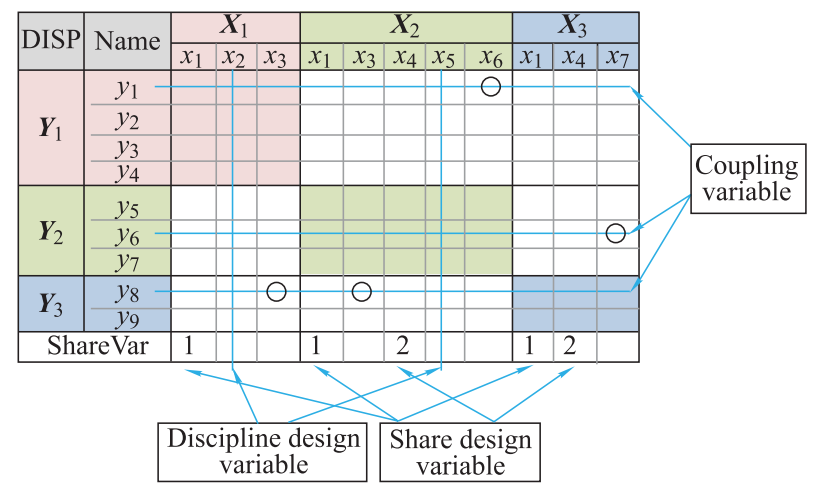

Fig. 3 Principle of the relation conversion for a three discipline MDO

This improved MDO format simplifies the MDO modeling process by connecting the MDO definition with the disciplinary input and output variables. Designers do not need to know the professional knowledge on MDO and avoid defining MDO variables in user's code $[19,20]$. It is very useful for designers without any MDO knowledge to optimize a complex MDO problem. Meanwhile, its standard conversion rule makes it easy to program, which will be explained in Section 4.

A small problem of the MDO modeling mentioned above is the variable repeatability due to the interface of disciplinary input/output variables. It will be confused for designers at first due to the several copies of share design variables and behavior variables in the objective function and constraints. Even these variables could be different in some MDO iterations for the unconvergence reason. In practice, these differences will fade away during the convergence procedure. Therefore, we should use one of them fixedly to avoid conflicts.

\section{MDO solving by DRM}

A considerable amount of researches have been done during the last decade for the MDO framework. There are so many useful commercial and open source MDO platforms, such as ISight, ModelCenter, VisualDOC, OpenM-
DAO, and CEASIOM. However, these frameworks do not pay much attention to the automation of MDO modeling and solving procedure. It is difficult to set up MDO architectures by these frameworks, and the costing time is also unacceptable. PyMDO [19-21] is a successful practice for the automation of MDO solving, but the nonintuitive script and complex problem definition are daunting for designers.

This section provides a brief introduction of some common MDO architectures with DRM. Based on these, the universal MDO organization and solving procedure are provided to explain the detailed solving approach.

\subsection{MDO architecture definition}

Martins provided a detailed survey of all available MDO architectures and gave a classification of these architectures into monolithic architecture and distributed architecture based on their problem formulations and decomposition strategies [22]. Following his definition, five MDO architectures are provided in the section, incuding individual discipline feasible (IDF), multidisciplinary feasible (MDF), concurrent subspace optimization (CSSO), collaborative optimization (CO) and analytical target cascading (ATC). These MDO architectures and MDO variables respected to the MDO definition with input/output variables are explained in the following.

\subsubsection{IDF architecture}

IDF is the simplest monolithic architecture. An MDO problem can be converted to an IDF architecture simply by defining some coupling variable copies as optimization variables and adding the related consistent constraints to the optimization problem. This may result in larger optimization problems to increase solving difficulties. The optimization problem with the input/output variables of the IDF architecture is

$$
\begin{gathered}
\min f(\boldsymbol{x}, \boldsymbol{y}(\boldsymbol{x}, \widehat{\boldsymbol{y}})) \\
\text { w.r.t. } \boldsymbol{x}, \widehat{\boldsymbol{y}} \\
\text { s.t. } h_{0}(\boldsymbol{x}, \boldsymbol{y}(\boldsymbol{x}, \widehat{\boldsymbol{y}}))=0 \\
h_{i}\left(\boldsymbol{x}_{0}, \boldsymbol{x}_{i}, \boldsymbol{y}_{i}\left(\boldsymbol{x}_{0}, \boldsymbol{x}_{i}, \widehat{\boldsymbol{y}}_{j \neq i}\right)\right)=0 \\
g_{0}(\boldsymbol{x}, \boldsymbol{y}(\boldsymbol{x}, \widehat{\boldsymbol{y}})) \leqslant 0 \\
g_{i}\left(\boldsymbol{x}_{0}, \boldsymbol{x}_{i}, \boldsymbol{y}_{i}\left(\boldsymbol{x}_{0}, \boldsymbol{x}_{i}, \widehat{\boldsymbol{y}}_{j \neq i}\right)\right) \leqslant 0 \\
c_{i}=\widehat{\boldsymbol{y}}_{i}-\boldsymbol{y}_{i}\left(\boldsymbol{x}_{0}, \boldsymbol{x}_{i}, \widehat{\boldsymbol{y}}_{j \neq i}\right)=0 \\
\boldsymbol{x}_{0}=\operatorname{Share}(\boldsymbol{X}) \\
\boldsymbol{x}_{i}=\operatorname{Sub}\left(\boldsymbol{X}_{i}\right), \quad \boldsymbol{y}_{i}=\operatorname{Couple}\left(\boldsymbol{Y}_{i}\right) \\
\boldsymbol{Y}_{i}=\operatorname{Disp}_{i}\left(\boldsymbol{X}_{i}\right) \\
i=1,2, \ldots, N
\end{gathered}
$$

where $h_{0}$ and $g_{0}$ are the equality constraints and nonequality constraints in the system level of the MDO problem, 
$h_{i}$ and $g_{i}$ are the equality constraints and nonequality constraints in the discipline level of the MDO problem. $\widehat{\boldsymbol{y}}$, the coupling variable copies, sometimes called target variables, is used to allow discipline analyses to run independently and in parallel. The conversion between the MDO variables and the discipline input/output variables are illustrated in Table 1.

\subsubsection{MDF architecture}

MDF is the smallest monolithic architecture. It satisfies the consistent constraints by the multidisciplinary analysis (MDA) process, which may cost extra time for convergence. The MDA process can be solved by fixed point iteration or Newton-based methods. However, the MDA process can be time-consuming and hard to parallel. The optimization problem with the input/output variables of the MDF architecture is

$$
\begin{gathered}
\min \quad f(\boldsymbol{x}, \boldsymbol{y}(\boldsymbol{x}, \boldsymbol{y})) \\
\text { w.r.t. } \boldsymbol{x} \\
\text { s.t. } h_{0}(\boldsymbol{x}, \boldsymbol{y}(\boldsymbol{x}, \boldsymbol{y}))=0 \\
h_{i}\left(\boldsymbol{x}_{0}, \boldsymbol{x}_{i}, \boldsymbol{y}_{i}\left(\boldsymbol{x}_{0}, \boldsymbol{x}_{i}, \widehat{\boldsymbol{y}}_{j \neq i}\right)\right)=0 \\
g_{0}(\boldsymbol{x}, \boldsymbol{y}(\boldsymbol{x}, \widehat{\boldsymbol{y}})) \leqslant 0 \\
g_{i}\left(\boldsymbol{x}_{0}, \boldsymbol{x}_{i}, \boldsymbol{y}_{i}\left(\boldsymbol{x}_{0}, \boldsymbol{x}_{i}, \widehat{\boldsymbol{y}}_{j \neq i}\right)\right) \leqslant 0 \\
\operatorname{MDA}_{(\boldsymbol{x})=0} \\
\boldsymbol{x}_{0}=\operatorname{Share}(\boldsymbol{X}) \\
\boldsymbol{x}_{i}=\operatorname{Sub}\left(\boldsymbol{X}_{i}\right), \quad \boldsymbol{y}_{i}=\operatorname{Couple}\left(\boldsymbol{Y}_{i}\right) \\
\boldsymbol{Y}_{i}=\operatorname{Disp}{ }_{i}\left(\boldsymbol{X}_{i}\right) \\
i=1,2, \ldots, N .
\end{gathered}
$$

The conversion between the MDO variables and the discipline input/output variables are illustrated in Table 1.

\subsubsection{CSSO architecture}

CSSO is a distributed architecture with a two-level optimization problem. It decomposes the MDO problem into some independent sub-problems to allow the parallel solution. CSSO develops a variety of improved forms by applying the approximation method and sensitivity information. The CSSO with the response surface (CSSO-RS) has fewer computational requirements by introducing the approximation method, which is more appropriate for complex engineering optimization problems. The couple variables of the discipline are surrogated to obtain the independent execution environment. The system sub-problem with the input/output variables of the CSSO-RC architecture is

$$
\min f(\boldsymbol{x}, \widetilde{\boldsymbol{y}}(\boldsymbol{x}, \widetilde{\boldsymbol{y}}))
$$

$$
\begin{gathered}
\text { w.r.t. } \boldsymbol{x} \\
\text { s.t. } h_{0}(\boldsymbol{x}, \widetilde{\boldsymbol{y}}(\boldsymbol{x}, \widetilde{\boldsymbol{y}}))=0 \\
h_{i}\left(\boldsymbol{x}_{0}, \boldsymbol{x}_{i}, \widetilde{\boldsymbol{y}}_{i}\left(\boldsymbol{x}_{0}, \boldsymbol{x}_{i}, \widetilde{\boldsymbol{y}}_{j \neq i}\right)\right)=0 \\
g_{0}(\boldsymbol{x}, \widetilde{\boldsymbol{y}}(\boldsymbol{x}, \widetilde{\boldsymbol{y}})) \leqslant 0 \\
g_{i}\left(\boldsymbol{x}_{0}, \boldsymbol{x}_{i}, \widetilde{\boldsymbol{y}}_{i}\left(\boldsymbol{x}_{0}, \boldsymbol{x}_{i}, \widetilde{\boldsymbol{y}}_{j \neq i}\right)\right) \leqslant 0 \\
\boldsymbol{x}_{0}=\operatorname{Share}(\boldsymbol{X}) \\
\boldsymbol{x}_{i}=\operatorname{Sub}\left(\boldsymbol{X}_{i}\right) \\
i=1,2, \ldots, N .
\end{gathered}
$$

The discipline $i$ sub-problem with the input/output variables of the CSSO-RC architecture is

$$
\begin{gathered}
\min \left(\boldsymbol{x}, \boldsymbol{y}_{i}\left(\boldsymbol{x}_{i}, \widetilde{\boldsymbol{y}}_{j \neq i}\right), \widetilde{\boldsymbol{y}}_{j \neq i}\right) \\
\text { w.r.t. } \boldsymbol{x}_{0}, \boldsymbol{x}_{i} \\
\text { s.t. } h_{0}(\boldsymbol{x}, \widetilde{\boldsymbol{y}}(\boldsymbol{x}, \widetilde{\boldsymbol{y}}))=0 \\
h_{i}\left(\boldsymbol{x}_{0}, \boldsymbol{x}_{i}, \boldsymbol{y}_{i}\left(\boldsymbol{x}_{0}, \boldsymbol{x}_{i}, \widetilde{\boldsymbol{y}}_{j \neq i}\right)\right)=0 \\
h_{j}\left(\boldsymbol{x}_{0}, \widetilde{\boldsymbol{y}}_{j}\left(\boldsymbol{x}_{0}, \widetilde{\boldsymbol{y}}\right)\right)=0 \\
g_{0}(\boldsymbol{x}, \widetilde{\boldsymbol{y}}(\boldsymbol{x}, \widetilde{\boldsymbol{y}})) \leqslant 0 \\
g_{i}\left(\boldsymbol{x}_{0}, \boldsymbol{x}_{i}, \boldsymbol{y}_{i}\left(\boldsymbol{x}_{0}, \boldsymbol{x}_{i}, \widetilde{\boldsymbol{y}}_{j \neq i}\right)\right) \leqslant 0 \\
g_{j}\left(\boldsymbol{x}_{0}, \widetilde{\boldsymbol{y}}_{j}\left(\boldsymbol{x}_{0}, \widetilde{\boldsymbol{y}}\right)\right) \leqslant 0, \\
\boldsymbol{x}_{0}=\operatorname{Share}(\boldsymbol{X}) \\
\boldsymbol{x}_{i}=\operatorname{Sub}\left(\boldsymbol{X}_{i}\right), \quad \boldsymbol{y}_{i}=\operatorname{Couple}\left(\boldsymbol{Y}_{i}\right) \\
\boldsymbol{Y}_{i}=\operatorname{Disp}{ }_{i}\left(\boldsymbol{X}_{i}\right) \\
j=1, \ldots, N, \quad j \neq i
\end{gathered}
$$

where $\widetilde{\boldsymbol{y}}$ is the surrogate model of the coupling variables $\boldsymbol{y}$. The conversion between the MDO variables and the discipline input/output variables are illustrated in Table 1.

\subsubsection{CO architecture}

$\mathrm{CO}$ is a popular distributed architecture. $\mathrm{CO}$ architecture decomposes the MDO problem into some discipline optimization sub-problems, which is similar to the organizational form of the industrial department. Every discipline is autonomous and can be easily integrated into the MDO solving procedure. The objective function is minimized at the system level and the consistency is optimized at the discipline level. The system sub-problem with the input/output variables of the $\mathrm{CO}$ architecture is

$$
\begin{gathered}
\min \quad f\left(\boldsymbol{x}_{0}, \widehat{\boldsymbol{x}}_{1}, \ldots, \widehat{\boldsymbol{x}}_{N}, \widehat{\boldsymbol{y}}\right) \\
\text { w.r.t. } \boldsymbol{x}_{0}, \widehat{\boldsymbol{x}}_{1}, \ldots, \widehat{\boldsymbol{x}}_{N}, \widehat{\boldsymbol{y}} \\
\text { s.t. } h_{0}\left(\boldsymbol{x}_{0}, \widehat{\boldsymbol{x}}_{1}, \ldots, \widehat{\boldsymbol{x}}_{N}, \widehat{\boldsymbol{y}}\right)=0 \\
g_{0}\left(\boldsymbol{x}_{0}, \widehat{\boldsymbol{x}}_{1}, \ldots, \widehat{\boldsymbol{x}}_{N}, \widehat{\boldsymbol{y}}\right) \leqslant 0
\end{gathered}
$$




$$
\begin{gathered}
J_{i}^{*}=\left\|\widehat{\boldsymbol{x}}_{0 i}-\boldsymbol{x}_{0}\right\|_{2}^{2}+\left\|\widehat{\boldsymbol{x}}_{i}-\boldsymbol{x}_{i}\right\|_{2}^{2}+ \\
\left\|\widehat{\boldsymbol{y}}_{i}-\boldsymbol{y}_{i}\left(\widehat{\boldsymbol{x}}_{0 i}, \boldsymbol{x}_{i}, \widehat{\boldsymbol{y}}_{j \neq i}\right)\right\|_{2}^{2} \leqslant \varepsilon, \\
\boldsymbol{x}_{0}=\operatorname{Share}(\boldsymbol{X}) \\
\boldsymbol{x}_{i}=\operatorname{Sub}\left(\boldsymbol{X}_{i}\right), \quad \boldsymbol{y}_{i}=\operatorname{Couple}\left(\boldsymbol{Y}_{i}\right) \\
i=1,2, \ldots, N .
\end{gathered}
$$

The discipline $i$ sub-problem with the input/output variables of the $\mathrm{CO}$ architecture is

$$
\begin{array}{r}
\min J_{i}\left(\widehat{\boldsymbol{x}}_{0 i}, \boldsymbol{x}_{i}, \boldsymbol{y}_{i}\left(\widehat{\boldsymbol{x}}_{0 i}, \boldsymbol{x}_{i}, \widehat{\boldsymbol{y}}_{j \neq i}\right)\right) \\
\text { w.r.t. } \widehat{\boldsymbol{x}}_{0 i}, \boldsymbol{x}_{i}, \widehat{\boldsymbol{y}}_{i} \\
\text { s.t. } h_{i}\left(\widehat{\boldsymbol{x}}_{0 i}, \boldsymbol{x}_{i}, \boldsymbol{y}_{i}\left(\widehat{\boldsymbol{x}}_{0 i}, \boldsymbol{x}_{i}, \widehat{\boldsymbol{y}}_{j \neq i}\right)\right)=0 \\
g_{i}\left(\widehat{\boldsymbol{x}}_{0 i}, \boldsymbol{x}_{i}, \boldsymbol{y}_{i}\left(\widehat{\boldsymbol{x}}_{0 i}, \boldsymbol{x}_{i}, \widehat{\boldsymbol{y}}_{j \neq i}\right)\right) \leqslant 0 \\
\boldsymbol{x}_{i}=\operatorname{Sub}\left(\boldsymbol{X}_{i}\right), \quad \boldsymbol{y}_{i}=\operatorname{Couple}\left(\boldsymbol{Y}_{i}\right) \\
\boldsymbol{Y}_{i}=\operatorname{Disp}_{i}\left(\boldsymbol{X}_{i}\right)
\end{array}
$$

where $\varepsilon$ is the relaxation tolerance of the consistency. Simply setting $\varepsilon$ to 0 may cause that the constraint gradients of the system problem at an optimal solution are all zero vectors, which will slow down the convergence for the gradient-based optimization algorithm. Given a small positive fixed value to $\varepsilon$ is the simplest approach to mitigate this problem, but there are some more efficient ways, such as dynamic tolerance coefficient [24], which is used in this paper. The conversion between the MDO variables and the discipline input/output variables are illustrated in Table 1.

\subsubsection{ATC architecture}

ATC is a distributed architecture. ATC architecture relaxes the consistency by penalty relaxation, and decomposes the MDO problem into the system level sub-problem and discipline level sub-problem. The system sub-problem with the input/output variables of the ATC architecture is

$$
\begin{gathered}
\min f(\boldsymbol{x}, \widehat{\boldsymbol{y}})+\sum_{i=1}^{N} \Phi_{i}\left(\widehat{\boldsymbol{x}}_{0 i}-\boldsymbol{x}_{0}, \widehat{\boldsymbol{y}}_{i}-\boldsymbol{y}_{i}\left(\boldsymbol{x}_{0}, \boldsymbol{x}_{i}, \widehat{\boldsymbol{y}}\right)\right)+ \\
\Phi_{h 0}\left(h_{0}(\boldsymbol{x}, \widehat{\boldsymbol{y}})\right)+\Phi_{g 0}\left(g_{0}(\boldsymbol{x}, \widehat{\boldsymbol{y}})\right) \\
\text { w.r.t. } \boldsymbol{x}_{0}, \widehat{\boldsymbol{y}} \\
\boldsymbol{x}_{0}=\operatorname{Share}(\boldsymbol{X}) \\
\boldsymbol{x}_{i}=\operatorname{Sub}\left(\boldsymbol{X}_{i}\right), \quad \boldsymbol{y}_{i}=\operatorname{Couple}\left(\boldsymbol{Y}_{i}\right) \\
i=1,2, \ldots, N
\end{gathered}
$$

The discipline $i$ sub-problem with the input/output variables of the ATC architecture is

$$
\begin{gathered}
\min f\left(\widehat{\boldsymbol{x}}_{0 i}, \boldsymbol{x}_{i}, \boldsymbol{y}_{i}\left(\widehat{\boldsymbol{x}}_{0 i}, \boldsymbol{x}_{i}, \widehat{\boldsymbol{y}}_{j \neq i}\right), \widehat{\boldsymbol{y}}_{j \neq i}\right)+ \\
\Phi_{i}\left(\widehat{\boldsymbol{x}}_{0 i}-\boldsymbol{x}_{0}, \widehat{\boldsymbol{y}}_{i}-\boldsymbol{y}_{i}\left(\widehat{\boldsymbol{x}}_{0 i}, \boldsymbol{x}_{i}, \widehat{\boldsymbol{y}}_{j \neq i}\right)\right)+
\end{gathered}
$$

$$
\begin{array}{r}
\Phi_{h 0}\left(h_{0}\left(\widehat{\boldsymbol{x}}_{0 i}, \boldsymbol{x}_{i}, \boldsymbol{y}_{i}\left(\widehat{\boldsymbol{x}}_{0 i}, \boldsymbol{x}_{i}, \widehat{\boldsymbol{y}}_{j \neq i}\right), \widehat{\boldsymbol{y}}_{j \neq i}\right)\right)+ \\
\Phi_{g 0}\left(g_{0}\left(\widehat{\boldsymbol{x}}_{0 i}, \boldsymbol{x}_{i}, \boldsymbol{y}_{i}\left(\widehat{\boldsymbol{x}}_{0 i}, \boldsymbol{x}_{i}, \widehat{\boldsymbol{y}}_{j \neq i}\right), \widehat{\boldsymbol{y}}_{j \neq i}\right)\right) \\
\text { w.r.t. } \widehat{\boldsymbol{x}}_{0 i}, \boldsymbol{x}_{i} \\
\text { s.t. } h_{i}\left(\widehat{\boldsymbol{x}}_{0 i}, \boldsymbol{x}_{i}, \boldsymbol{y}_{i}\left(\widehat{\boldsymbol{x}}_{0 i}, \boldsymbol{x}_{i}, \widehat{\boldsymbol{y}}_{j \neq i}\right), \widehat{\boldsymbol{y}}_{j \neq i}\right)=0 \\
g_{i}\left(\widehat{\boldsymbol{x}}_{0 i}, \boldsymbol{x}_{i}, \boldsymbol{y}_{i}\left(\widehat{\boldsymbol{x}}_{0 i}, \boldsymbol{x}_{i}, \widehat{\boldsymbol{y}}_{j \neq i}\right), \widehat{\boldsymbol{y}}_{j \neq i}\right) \leqslant 0 \\
\boldsymbol{x}_{i}=\operatorname{Sub}\left(\boldsymbol{X}_{i}\right), \quad \boldsymbol{y}_{i}=\operatorname{Couple}\left(\boldsymbol{Y}_{i}\right) \\
\boldsymbol{Y}_{i}=\operatorname{Disp}_{i}\left(\boldsymbol{X}_{i}\right)
\end{array}
$$

where $\Phi_{i}, \Phi_{h 0}$ and $\Phi_{g 0}$ are the penalty function of the consistent constraints, system level equality constraints and system level nonequality constraints. The augmented Lagrangian function form is used here as follows:

$$
\begin{gathered}
\Phi_{i}=\boldsymbol{v}_{c}^{\mathrm{T}}\left(\boldsymbol{y}_{i}-\widehat{\boldsymbol{y}}_{i}\right)+\left\|\boldsymbol{w}_{c}\left(\boldsymbol{y}_{i}-\widehat{\boldsymbol{y}}_{i}\right)\right\|_{2}^{2} \\
\Phi_{h}=\boldsymbol{v}_{h}^{\mathrm{T}} h_{0}+\left\|\boldsymbol{w}_{h} h_{0}\right\|_{2}^{2} \\
\Phi_{g}=\boldsymbol{v}_{g}^{\mathrm{T}} g_{0}+\left\|\boldsymbol{w}_{g} g_{0}\right\|_{2}^{2}
\end{gathered}
$$

where $\boldsymbol{v}_{c}, \boldsymbol{v}_{h}$ and $\boldsymbol{v}_{g}$ are the Lagrangian multipliers of the augmented Lagrangian function. $\boldsymbol{w}_{c}, \boldsymbol{w}_{h}$ and $\boldsymbol{w}_{g}$ are the penalty weights of the augmented Lagrangian function. When $\boldsymbol{v}_{c}, \boldsymbol{v}_{h}$ and $\boldsymbol{v}_{g}$ are all set to be 0, (12) is degraded into the quadratic penalty function. $v$ is updated by the following equation,

$$
\begin{gathered}
\boldsymbol{v}^{k+1}=\boldsymbol{v}^{k}+2 \boldsymbol{w}^{k} \cdot \boldsymbol{w}^{k} \cdot \boldsymbol{q}^{k} \\
\boldsymbol{q}=\left[\begin{array}{l}
c \\
h_{0} \\
g_{0}+t_{0}^{2}
\end{array}\right]
\end{gathered}
$$

where $c$ is the consistent constraint. $t_{0}$ is the relaxation factor of the nonequality constraints. The following updated strategy is used to update $t_{0}$,

$$
\boldsymbol{w}_{i}^{k+1}= \begin{cases}\boldsymbol{w}_{i}^{k}, & \left|\boldsymbol{q}_{i}^{k}\right| \leqslant \gamma\left|\boldsymbol{q}_{i}^{k-1}\right| \\ \beta \boldsymbol{w}_{i}^{k}, & \left|\boldsymbol{q}_{i}^{k}\right|>\gamma\left|\boldsymbol{q}_{i}^{k-1}\right|\end{cases}
$$

where $k$ is the current iteration step of the outer loop. $\beta>1,0<\gamma<1$. The conversion between the MDO variables and the discipline input/output variables are illustrated in Table 1.

\subsection{MDO solving procedure}

The detail coupling relationship of an MDO problem can be represented by the DRM based on the disciplinary input and output variables. Making use of its advantages, the MDO solving procedure could be decomposed into three parts: the DRM coupling relationship definition, the MDO problem description based on disciplinary input/output variables and the MDO solving process executed automatically based on MDO architectures. The function of each part is explained as 
(i) DRM coupling relationship definition

By analyzing the DRM, the coupling relationship between MDO variables and disciplinary input/output variables can be recognized based on the principle in Table 1 . Also we can find the numbers of the disciplines, the dimensions and the sequence of the input/output variables of each discipline.

(ii) MDO problem description

The system level objective function, system level constraints and discipline level constraints are constructed based on the disciplinary input and output variables.

(iii) MDO solving

The MDO problem is converted into some optimization problems based on the executive structure of the MDO architectures and solved by the optimization algorithm.

The procedure of MDO solving based on the DRM is shown as Fig. 4. For an MDO problem with $N$ disciplines, the detail implementation is as follows:

Step 1 Building the DRM

Build the DRM based on the disciplinary input/output variables, mark the coupling relationship if the output variable of one discipline is connected to the input variable of another discipline, and then select the share design variable. Then we have the full coupling relationship with the input/output variables based on the rules on Table 1.

Step 2 Reconstructing the MDO problem

Based on (3), the system level objective function, system level constraints and discipline level constraints are defined with disciplinary input and output variables, set the boundaries of disciplinary input/output variables and the constraints, and then wrap the discipline models into standard input/output interfaces [5].

Step 3 Decomposing and reconstructing MDO system

Depending on the coupling relationship defined by the DRM and the MDO problem description, decompose and reconstruct the MDO system. Based on the MDO architectures introduced in Section 3.1, convert the MDO problem into some optimization problems with the help of the surrogate model, optimization algorithm and sensitivity analysis.

Step 4 Solving MDO problem

Build convergence criterion and solve the above established MDO problem according to the process of different MDO architectures.

The procedure of MDO solving is programed as some $\mathrm{C}++$ Classes. A brief software design introduction is illustrated in Section 4.

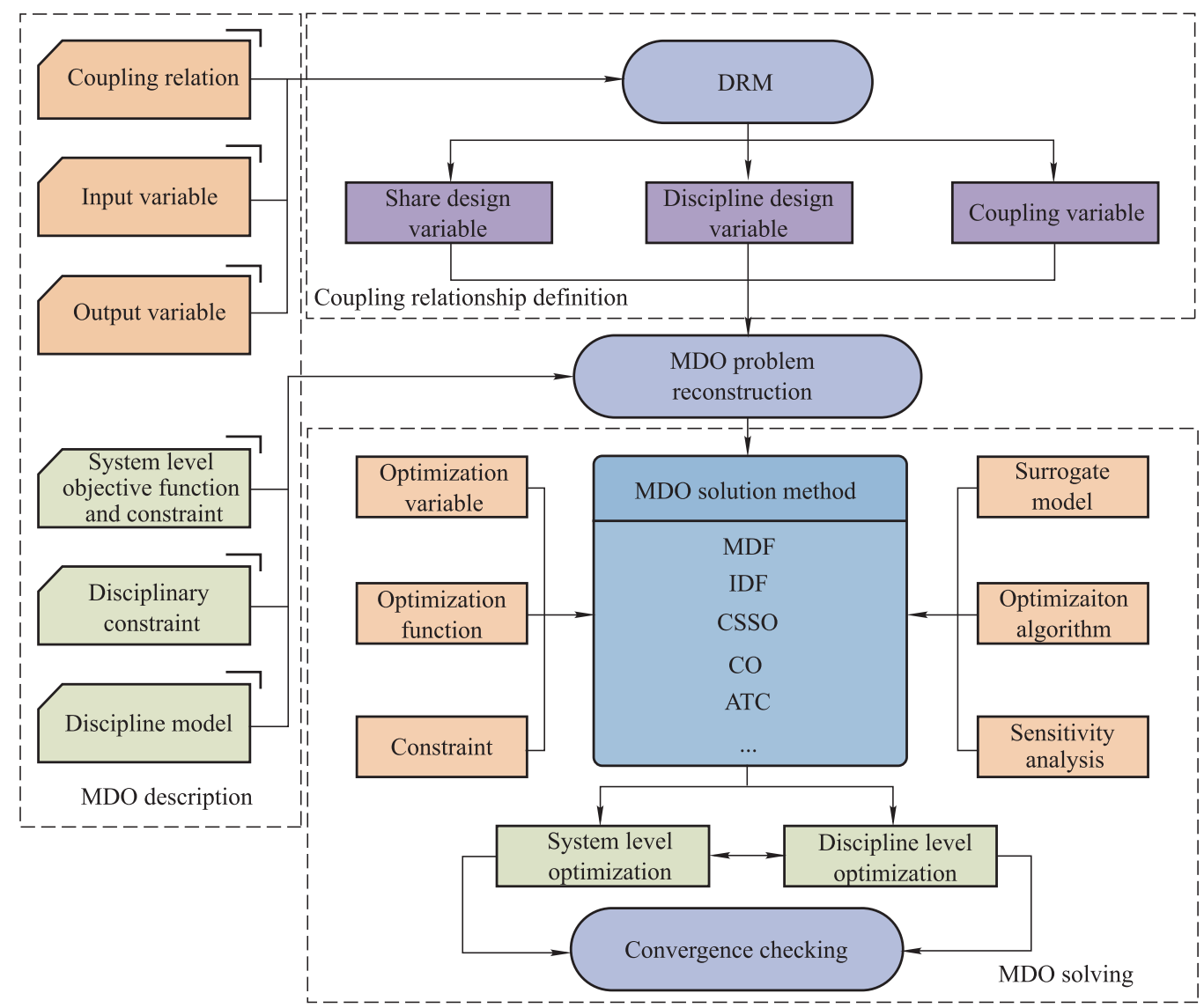

Fig. 4 Procedure of MDO solving based on DRM 


\section{Software design}

An object-oriented framework is established in C++ environment to the modularized and automated way for MDO modeling and solving, which is called as automatic fast solving environment for MDO (AFSEMDO). The framework is designed as an automatic solving environment. Once an MDO problem has been imported into AFSEMDO with the proper format and some solving parameters are setup, e.g. the type of MDO architecture, the type of optimization algorithm, convergence precision, no additional work is required during the MDO modeling and solving process. This is achieved by a modularized and universal implementation procedure which is detailed introduced as follows.

The MDO solving environment includes the following modules: MDO solving kernel, decomposition strategy, optimization algorithm, approximation method, subsystem, sub-discipline, discipline wrapper, coupling relationship and MDO problem description. The modules of the optimization algorithm, approximation method and discipline wrapper are the generic components supported by the Mcdesign [24] framework, these are designed as Dynamic Link Library (DLL) which can be easily integrated into ASMEMDO. The sub-discipline module manages the discipline model and the sub-system manages the converted discipline model. The discipline model here means the real discipline model containing the complete disciplinary input and output variables which come from the discipline wrapper. The converted discipline model here means the standard discipline model containing the shared design variables, discipline design variables and coupling variables. These MDO variables can be directly used by the MDO architecture. The sub-system is used as the interfaces between the discipline analysis code and the MDO solving environment to standardize and simplify the original discipline model. The decomposition strategy module is designed as the standard execution flow for the decomposition strategies, for a given MDO problem with disciplinary input/output interfaces, it will be converted into some optimization problems and be coded into standard execution process. Coupling relationship module is responsible for the conversion between disciplinary input/output variables and MDO variables based on the principle in Table 1 . The MDO solving kernel is the entry of the MDO modeling and solving, it manages all the modules mentioned above and executes the solving process automatically by the given configuration. The MDO problem description module inputs the basic MDO definition, the objective function and constraints of the optimization problem and the DRM matrix information. The relation di- agram of these modules is listed in Fig. 5.

A base class is defined as follows to give a general definition of the MDO problem structure to the AFSEMDO framework.

class BaseMDOProblem

\{

public:

BaseMDOProblem(void) \{\}

virtual $\sim$ BaseMDOProblem(void) \{\}

// Problem information

virtual bool get $\_$mdo info(string \& mdoname, int\& disp-

num) $=0$;

virtual bool get_drm_info(string \& drmname $)=0$;

virtual bool get_disp_info(int idisp, string\& dispname, vector $<$ Var $>\&$ inputvar, vector $<$ Var $>\&$ outputvar $)=0$;

virtual bool get_disp_fun_con_info(int idisp,Var\& dispfun, vector $<$ Var $>\&$ dispcon $)=0$;

virtual bool get_sys_fun_con_info(Var\& sysfun, vector $<$ Var $>$ \& syscon $)=0$;

\section{// Evaluation}

virtual bool eval_disp_fun(int idisp, vector $<$ Var $>$ inputvar, vector $<$ Var $>$ \& outputvar $)=0$;

virtual bool eval_disp_obj_con(int idisp, vector $<$ Var $>$ inputvar, vector $<$ Var $>$ outputvar, Var\& dispobj, vector $<$ Var $>\&$ dispcon $)=0$;

virtual bool eval_sys_obj_con(vector $<$ vector $<$ Var $>>$ inputvars, vector $<$ vector $<$ Var $>>$ outputvars, Var\& sysobj, vector $<$ Var $>\&$ syscon $)=0$;

// Result

virtual void finalize_solution(int status, MDOIterHistory last) $=0$;

virtual bool intermediate_callback(int status, int iter, MDOIterHistory cur $)=0$;

private:

BaseMDOProblem(const BaseMDOProblem\&);

void operator $=($ const BaseMDOProblem\& $)$; \} ;

The definition of the class BaseMDOProblem can be divided into three types: problem information, evaluation and result. Problem information setups the name of the MDO problem and the numbers of discipline by the function get $m$ do info, loads the DRM information by the function get_drminfo; gets the information of the input and output variables of disciplines by the function get_disp_info, and also gets the information of the objective function and constraints of the system level and discipline level by the functions get sys fun _con info and get_disp_fun_con_info. Evaluation defines the execution functions of the discipline model by the function eval_disp_fun, and also defines the objective function and 


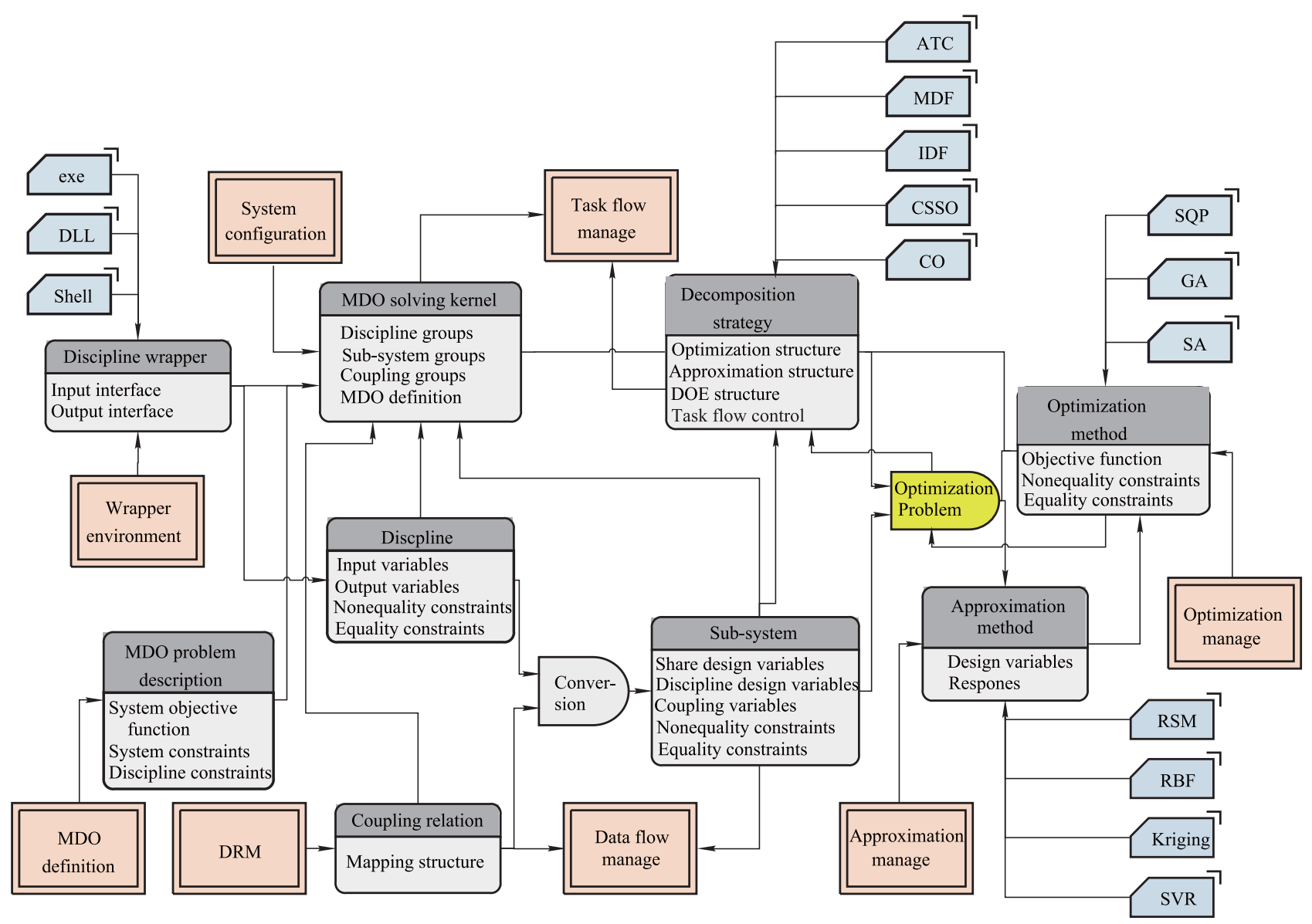

Fig. 5 Relationship of the modules in AFSEMDO

constraints of the system level and discipline level by the functions eval_sys_obj_con and eval_disp_obj_con. The result gives some interfaces to get the final solution and the result of every calculation steps by the function of finalize_solution and intermediate_callback. Var is the standard definition of the variable including the name, initialization, boundary and current value, which is used as the communication interfaces among these modules in AFSEMDO. MDOIterHistory contains the history of the total MDO iteration. Once an MDO problem is inherited from the standard BaseMDOProblem class, it can be solved by the AFSEMDO framework automatically.

\section{Examples}

\subsection{Reusable launch vehicle MDO modeling}

Reusable launch vehicle (RLV) is one of the development trends of the future launch vehicle. It is a complex multidisciplinary problem, which should make consideration of the aerodynamic, structure, trajectory, layout, thermal and control system at the conceptual design phase. A typical
RLV problem is used to show an example about how to generate an MDO problem by DRM.

Five disciplines are considered in the RLV problem, including aerodynamic, structure, trajectory, layout and thermal. The aerodynamic discipline calculates the aerodynamic data and geometry measurement, taking geometry parameters as input variables; the structure discipline calculates the structure mass and structural strength, taking geometry parameters, structure size parameters, aerodynamic data from aerodynamic discipline and trajectory data from trajectory discipline as input variables; thermal discipline calculates TPS mass and material distribution, taking geometry parameters, TPS thickness, trajectory data from trajectory discipline and aerodynamic data from aerodynamic discipline as inputs variables; layout discipline calculates layout configuration and mass distribution, taking structure mass from structure discipline, TPS mass from thermal discipline, fuel mass from trajectory discipline as input variables; trajectory discipline calculates fuel mass and trajectory path data, taking control data, aerodynamic data from aerodynamic discipline, and dry mass 
from the layout discipline as input variables. These couplings make a complex RLV MDO problem, which can be shown by the DRM in Fig. 6. Each variable in the DRM means a variable set. For example, geometry variables may include body length, body width, and wing span.

The MDO problem of RLV is generated based on the DRM modeling method. The following MDO variables can be extracted from DRM of the RLV problem in Table 2. From Fig. 6 we can find, geometry variables are the only one share design variable in ShareVar row. Structure variables, TPS thickness and control data are with no coupling from other outputs variables (There are no circle marks in the crossed element in the DRM), so these variables are discipline design variables. The other input variables are connected with output variables from other disciplines (There are circle marks), so these variables are coupling variables and the coupling relationship can be easily found in the corresponding position of DRM.

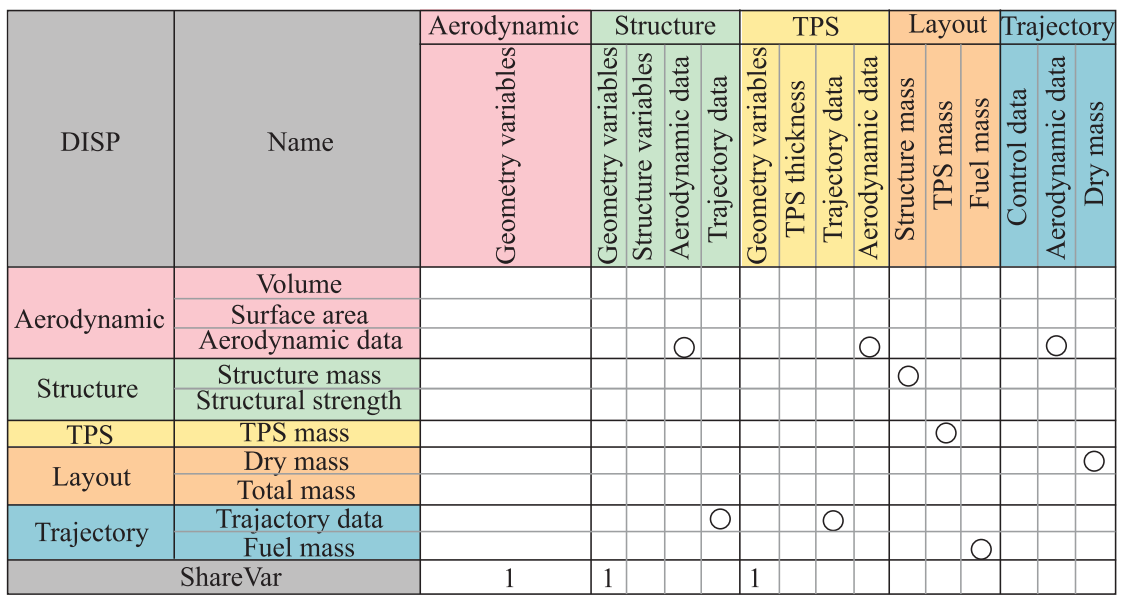

Fig. 6 DRM of the RLV problem

Table 2 MDO variables of RLV problem based on DRM

\begin{tabular}{cc}
\hline MDO variable & Definition \\
\hline $\begin{array}{c}\text { Discipline design } \\
\text { variable }\end{array}$ & $\begin{array}{c}\text { Structure variables (structure discipline) } \\
\text { TPS thickness (TPS discipline) } \\
\text { Control data (trajectory discipline) }\end{array}$ \\
\hline Share design variable & Geometry variables \\
\hline & Aerodynamic data (from aerodynamic \\
discipline)
\end{tabular}

Objective function and constraints can be any combinations of the input/output variables of these disciplines. Total mass is selected as the objective function. Dry mass and volume are selected as the constraints. The following MDO model based on IDF architecture can be generated using the automatic MDO solving procedure introduced in Section 3. Other MDO architectures are similar.

\section{min Total mass}

w.r.t. Geometry variables, Structure variables, TPS thickness, Control data, Trajectory data,

Aerodynamic data, Structure mass, TPS mass, Fuel mass, Dry mass s.t. Dry mass $\leqslant M_{1}$ Volume $\geqslant V_{1}$

Aerodynamic data - Aerodynamic data ${ }^{*}=0$

Trajectory data - Trajectory data ${ }^{*}=0$

Structure mass - Structure mass ${ }^{*}=0$

TPS mass - TPS mass* $=0$

Fuel mass - Fuel mass ${ }^{*}=0$

Dry mass - Dry mass ${ }^{*}=0$

where ()$^{*}$ means the variables form optimizer.

\subsection{MDO benchmarks testing}

SNOPT [25] is used as the system and subsystem's optimization solver. This is based on the sequential quadratic programming (SQP) algorithm with many common interfaces. The sensitivities of the objective function and constraints with respect to the optimization variables are got from the finite differencing method. Radial basis function (RBF) is used as the surrogate model. And the Latin hypercube method is used as the sampling method in AFSEMDO.

\subsubsection{Benchmarks}

Three benchmarks are used to test the MDO modeling and solving framework based on the DRM. The first one is an 
analytic example that has been previously solved by Sellar [26] et al. It is a two disciplines MDO problem and has low dimensionality. The second is adapted from NASA's MDO test suite [27] and represents the design of a simple gearbox. As the problem has originally been solved as a single discipline optimization problem, the MDO problem is created by splitting up the single discipline problem into gear 1, gear 2 and gear shaft three parts with no coupling. The third example is a complex aircraft problem with aerodynamic, mass and performance disciplines. Detail information and results can be found in [5]. The three MDO benchmarks are listed as follows:

(i) Analytic problem

$$
\begin{gathered}
\min \quad x_{2}^{2}+x_{3}+y_{1}+e^{-y_{1}} \\
\text { w.r.t. } \quad x_{1}, x_{2}, x_{3} \\
\text { s.t. } \quad 1-y_{1} / 3.16 \leqslant 0 \\
y_{2} / 24-1 \leqslant 0 \\
-10 \leqslant x_{1} \leqslant 10 \\
0 \leqslant x_{2} \leqslant 10 \\
0 \leqslant x_{3} \leqslant 10
\end{gathered}
$$

Discipline 1,

Given $x_{1}, x_{2}, x_{3}, y_{2}$

$$
\begin{gathered}
y_{1}=x_{1}^{2}+x_{2}+x_{3}-0.2 y_{2} \\
\text { Return } y_{1}
\end{gathered}
$$

Discipline 2,

$$
\begin{gathered}
\text { Given } x_{1}, x_{3}, y_{1} \\
y_{2}=\sqrt{y_{1}}+x_{1}+x_{3} \\
\text { Return } y_{2}
\end{gathered}
$$

The DRM of analytic problem is shown in Fig. 7.

\begin{tabular}{|c|c|c|c|c|c|c|c|c|}
\hline \multirow{2}{*}{ DISP } & \multirow{2}{*}{ Name } & \multicolumn{4}{|c|}{$\boldsymbol{X}_{1}$} & \multicolumn{3}{c|}{$\boldsymbol{X}_{2}$} \\
\hline & & $x_{1}$ & $x_{2}$ & $x_{3}$ & $y_{2}$ & $x_{1}$ & $x_{3}$ & $y_{1}$ \\
\hline $\boldsymbol{Y}_{1}$ & $y_{1}$ & & & & & & & $\bigcirc$ \\
\hline $\boldsymbol{Y}_{2}$ & $y_{2}$ & & & & $\bigcirc$ & & & \\
\hline \multicolumn{2}{|c|}{ ShareVar } & 1 & & 2 & & 1 & 2 & \\
\hline
\end{tabular}

Fig. 7 DRM of analytic problem

(ii) Golinski's speed reducer

$\min C_{1} x_{1} x_{2}^{2}\left(C_{2} x_{3}^{2}+C_{3} x_{3}-C_{4}\right)-C_{5} x_{1}\left(x_{6}^{2}+x_{7}^{2}\right)+$

$$
\begin{gathered}
C_{6}\left(x_{6}^{3}+x_{7}^{3}\right)+C_{7}\left(x_{4} x_{6}^{2}+x_{5} x_{7}^{2}\right) \\
\text { w.r.t. } x_{1}, x_{2}, x_{3}, x_{4}, x_{5}, x_{6}, x_{7}
\end{gathered}
$$

\begin{tabular}{|c|c|c|c|c|c|c|c|c|c|c|c|c|}
\hline \multirow{2}{*}{ DISP } & \multirow{2}{*}{ Name } & \multicolumn{4}{|c|}{$\boldsymbol{X}_{1}$} & \multicolumn{4}{|c|}{$X_{2}$} & \multicolumn{3}{|c|}{$X_{3}$} \\
\hline & & $x_{2}$ & $x_{3}$ & $x_{4}$ & $x_{6}$ & $x_{2}$ & $x_{3}$ & $x_{5}$ & $x_{7}$ & $x_{1}$ & $x_{2}$ & $x_{3}$ \\
\hline \multirow{3}{*}{$\boldsymbol{Y}_{1}$} & $g_{3}$ & & & & & & & & & & & \\
\hline & $g_{5}$ & & & & & & & & & & & \\
\hline & $g_{10}$ & & & & & & & & & & & \\
\hline \multirow{3}{*}{$\boldsymbol{Y}_{2}$} & $g_{4}$ & & & & & & & & & & & \\
\hline & $g_{6}$ & & & & & & & & & & & \\
\hline & $g_{11}$ & & & & & & & & & & & \\
\hline \multirow{5}{*}{$\boldsymbol{Y}_{3}$} & $g_{1}$ & & & & & & & & & & & \\
\hline & $g_{2}$ & & & & & & & & & & & \\
\hline & $g_{7}$ & & & & & & & & & & & \\
\hline & $g_{8}$ & & & & & & & & & & & \\
\hline & $g_{9}$ & & & & & & & & & & & \\
\hline \multicolumn{2}{|c|}{ Share Var } & 1 & 2 & & & 1 & 2 & & & & 1 & 2 \\
\hline
\end{tabular}

Discipline 1,

Given $x_{2}, x_{3}, x_{4}, x_{6}$

$$
g_{3}, g_{5}, g_{10} \leqslant 0
$$

Discipline 2,

$$
\begin{gathered}
\text { Given } x_{2}, x_{3}, x_{5}, x_{7} \\
g_{4}, g_{6}, g_{11} \leqslant 0
\end{gathered}
$$

Discipline 3,

$$
\begin{gathered}
\text { Given } x_{1}, x_{2}, x_{3} \\
g_{1}, g_{2}, g_{7}, g_{8}, g_{9} \leqslant 0
\end{gathered}
$$

The DRM of Golinski's speed reducer is shown in Fig. 8.

Fig. 8 DRM of Golinski's speed reducer

(iii) Aircraft problem

$$
\min W_{t o}
$$

$$
\begin{gathered}
\text { s.t. } \quad R \geqslant 901.23 \mathrm{~km} \\
V_{s} \leqslant 21.336 \mathrm{~m} / \mathrm{s}
\end{gathered}
$$

Discipline 1,

Given $A R, S_{w}, L_{f}, D_{f}$

$$
\begin{gathered}
L / D=10.0+4.0\left(\frac{A R}{S_{\text {wet }} / S_{w}}-1\right) \\
S_{\text {wet }}=S_{\text {fuse }}+2 S_{w}+3 \times 0.2 S_{w} \\
S_{\text {fuse }}=\pi L_{f} D_{f}
\end{gathered}
$$

Return $L / D$

Discipline 2,

Given $L_{f}, D_{f}, \rho_{c}, V_{v}, L / D, W_{\text {fuel }}$

$$
W_{\text {to }}=3\left(W_{\text {fuse }}+W_{\text {fuel }}\right)
$$

$W_{\text {fuse }}=2.862 L_{f}^{1.035} D_{f}^{1.086} \rho_{c}^{0.241} V_{c}^{0.482}(L / D)^{-0.072}$

Return $W_{\text {to }}$ 
Discipline 3,

$$
\begin{gathered}
\text { Given } L / D, W_{\mathrm{to}}, W_{\text {fuel }}, \rho_{c}, S_{w} \\
R=\eta \frac{L / D}{c} \ln \left(\frac{W_{\mathrm{to}}}{W_{\mathrm{to}}-0.85 W_{\text {fuel }}}\right) \\
V_{s}=\sqrt{\frac{2 W_{t o}}{\rho_{c} S_{w} C_{L \max }}}
\end{gathered}
$$

Return $R, V_{s}$

The DRM of the aircraft problem is shown in Fig. 9.

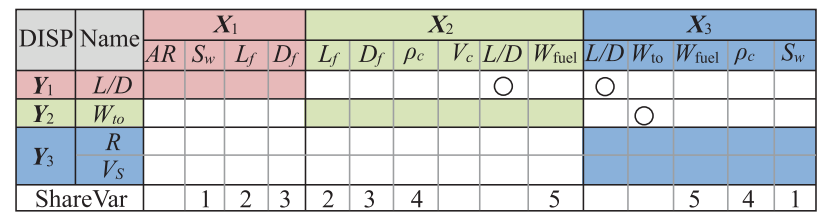

Fig. 9 DRM of aircraft problem

\subsubsection{Results}

Five MDO architectures are tested in the framework AFSEMDO based on the DRM method proposed in this article. The results of the above three benchmarks are listed in

\begin{tabular}{|c|c|c|c|c|c|c|}
\hline \multirow{2}{*}{ Benchmark } & \multirow{2}{*}{$\begin{array}{c}\text { MDO } \\
\text { architecture }\end{array}$} & \multicolumn{3}{|c|}{ The number of discipline evaluation } & \multicolumn{2}{|c|}{ Objective function } \\
\hline & & Discipline 1 & Discipline 2 & Discipline 3 & Optimal value & Relative error \\
\hline \multirow{5}{*}{ Case 1} & MDF & 203 & 203 & - & 3.18341 & $5.2339 \mathrm{E}-06$ \\
\hline & IDF & 139 & 139 & - & 3.18339 & $2.4801 \mathrm{E}-06$ \\
\hline & CSSO & 339 & 432 & - & 3.1834 & $3.2731 \mathrm{E}-06$ \\
\hline & $\mathrm{CO}$ & 6589 & 2982 & - & 3.1835 & $5.3615 \mathrm{E}-05$ \\
\hline & ATC & 10475 & 5769 & - & 3.1833 & $2.8802 \mathrm{E}-05$ \\
\hline \multirow{5}{*}{ Case 2} & MDF & 73 & 73 & 73 & 2994.351 & $1.3349 \mathrm{E}-06$ \\
\hline & IDF & 73 & 73 & 73 & 2994.351 & $1.3349 \mathrm{E}-06$ \\
\hline & CSSO & 773 & 1021 & 1071 & 2994.355 & 7.709 9E-06 \\
\hline & $\mathrm{CO}$ & 14467 & 14812 & 11139 & 2994.547 & $6.4127 \mathrm{E}-05$ \\
\hline & ATC & 3269 & 3090 & 1456 & 2994.361 & $2.0038 \mathrm{E}-06$ \\
\hline \multirow{5}{*}{ Case 3} & MDF & 638 & 638 & 638 & 875.6268 & $3.1381 \mathrm{E}-05$ \\
\hline & IDF & 213 & 213 & 213 & 875.6537 & $5.9494 \mathrm{E}-07$ \\
\hline & CSSO & 5667 & 5401 & 6815 & 875.6535 & 3.945 5E-06 \\
\hline & $\mathrm{CO}$ & 15009 & 182326 & 15957 & 875.1605 & 0.00055907 \\
\hline & ATC & 2280 & 7642 & 4818 & 955.4582 & 0.091142 \\
\hline
\end{tabular}
Table 3.

Table 3 Results of the three benchmarks

All benchmarks have converged except the third case with ATC. As you can see from Table 3, the monolithic architectures such as MDF and IDF have a steady convergence procedure and high computation efficiency. Distributed architectures such as CSSO, ATC and CO, have to take more time to converge to the optimal solution. These results obtained are in agreement with the results in $[20,28]$, which give a good proof that the DRM conversion has no additional effect on the results of the MDO modeling and solving.

From the number of discipline evaluation and the objective function relative error, we come to a conclusion that the monolithic architectures have a better performance than the distributed architectures beside the parallel computing environment. Although the process of conversion could add some additional cost, considering the timeconsuming process of the discipline evaluation, it could be negligible in the MDO solving procedure.

The results of these three benchmarks show the framework AFSEMDO has well supported for the MDO modeling and solving. The MDO model based on DRM finds out the relationship between the MDO variables and disciplinary input/output variables conveniently, and it emphasizes the importance of the discipline model, which is the basic element of the MDO problem. Especially for blackbox discipline model, the disciplinary input and output interfaces of which are the only information could be got. The complex original MDO definition has been converted to the new MDO formation based on DRM, so the MDO architectures are untied from the MDO model and can be used with no additional modification to any MDO problem, which make it possible for the automation and modularization.

\section{Conclusions and future work}

General formulation of the MDO problem is defined based on the DRM. The MDO modeling and solving methods according to the new formulation are presented. The RLV problem is used to illustrate the MDO modeling process and three benchmarks are tested in the new framework AFSEMDO. 
(i) The DRM based on disciplinary input and output variables offers a simple and visualized interface for MDO definition, and proposes an automatic and modularized way of MDO modeling and solving.

(ii) The new MDO solving procedure using the interface of disciplinary input and output variables is discussed in this paper, and the main idea is to create an automatic MDO environment for the industrial problems with blackbox disciplines.

(iii) Three benchmarks are tested and compared in the AFSEMDO. From these results, it can be concluded that the framework AFSEMDO with the DRM method could provide a more simplified way to solve MDO problems.

Further studies are needed to extend the DRM method to support MDO coupling definition considering uncertainty. Applying this DRM method to dynamic decomposing and reconstructing of the MDO problem deserves detailed study. The ability of automatic process also needs further research to establish a more efficient and convenient MDO framework.

\section{References}

[1] T. W. Simpson, J. R. R. A. Martins. The future of multidisciplinary design optimization (MDO): advancing the design of complex engineered systems. NSF Workshop Report, Fort Worth, Texas, 2010.

[2] Z. Yin, D. Mi, L. Wu, et al. A summary of MDO based high integrated performance aero-engine research program. Proc. of the 49th AIAA/ASME/ASCE/AHS/ASC Structures, Structural Dynamics, and Materials Conference, 2008.

[3] W. S. Timothy, T. Vassili, B. Vladimir, et al. Design and analysis of computer experiments in multidisciplinary design optimization: a review of how far we have come - or not. Proc. of the 12th AIAA/ISSMO Multidisciplinary Analysis and Optimization Conference, 2008.

[4] O. D. Weck, J. Agte, J. Sobieszczanski-Sobieski, et al. Stateof-the-art and future trends in multidisciplinary design optimization. Proc. of the 48th AIAA/ASME/ASCE/AHS/ASC Structures, Structural Dynamics, and Materials Conference, 2007.

[5] H. Su. Study on high fidelity multidisciplinary design optimization technique of vehicles. Xi' an: Northwestern Polytechnical University, 2014. (in Chinese)

[6] D. V. Steward. The design structure system: a method for managing the design of complex systems. IEEE Trans. on Engineering Management, 1981, EM-28(3): 71-74.

[7] T. R. Browning. Applying the design structure matrix to system decomposition and integration problems: a review and new directions. IEEE Trans. on Engineering Management, 2001, 48(3): 292-306.

[8] A. B. Lambe, J. R. R. A. Martins. Extensions to the design structure matrix for the description of multidisciplinary design, analysis, and optimization processes. Structural and Multidisciplinary Optimization, 2012, 46(2): 273 - 284.

[9] D. Tang, G. Zhang, S. Dai. Design as integration of axiomatic design and design structure matrix. Robotics and ComputerIntegrated Manufacturing, 2009, 25: 610-619.

[10] M. S. Avnet, A. L. Weigel. An application of the design struc- ture matrix to integrated concurrent engineering. Acta Astronautica, 2010, 66(5): $937-949$.

[11] D. Mike, R. B. Tyson. Managing complex product development projects with design structure matrices and domain mapping matrices. International Journal of Project Management, 2007, 25: 300-314

[12] T. L. Yu, D. E. Goldberg, K. Sastry, et al. Dependency structure matrix, genetic algorithms, and effective recombination. Evol Compute, 2009, 17(4): 595-626.

[13] S. K. Ramprasad, Y. P. Panos. Hierarchical decomposition synthesis in optimal systems design. Journal of Material Chemistry, 1997, 36(3-1): 494-498.

[14] S. Li. A matrix-based clustering approach for the decomposition of design problems. Research in Engineering Design, 2011, 22(4): $263-278$.

[15] K. A. Kusia, G. C. Chen. A branch-and-bound algorithm for solving the group technology problems. Annals of Operations Research, 1991, 26(26): 415-431.

[16] S. K. Ramprasad, Y. P. Panos. Optimal hierarchical decomposition synthesis using integer programming. Journal of Mechanical Design, 1995, 119(4): 440-447.

[17] L. Chen, Z. Ding, S. Li. Tree-based dependency analysis in decomposition and re-decomposition of complex design problems. Journal of Mechanical Design, 2005, 127(1): 12 - 23.

[18] H. Su, L. X. Gu, C. L. Gong. A disciplinary relation matrix based universal multidisciplinary optimization architecture. Computer Integrated Manufacturing Systems, 2014, 20(4): 731 - 738. (in Chinese)

[19] J. R. R. A. Martins, C. Marriage, N. Tedford. PyMDO: an object-oriented framework for multidisciplinary design optimization. ACM Transactions on Mathematical Software (TOMS), 2009, 36(4): 1-25.

[20] N. P. Tedford, J. R. R. A. Martins. Comparison of MDO architectures within a common python framework. Proc. of the 47th AIAA/ASME/ASCE/AHS/ASC Structures, Structural Dynamics, and Materials Conference, 2006.

[21] J. J. Alonso, P. LeGresley, E. Weide, et al. PyMDO - a framework for high-fidelity multi-disciplinary optimization. Proc. of the 10th AIAA/ISSMO Multidisciplinary Analysis and Optimization Conference, 2004.

[22] J. R. R. A. Martins. Multidisciplinary design optimization: a survey of architectures. AIAA Journal, 2013, 51(9): $2049-$ 2075.

[23] X. Li, W. Li, C. Liu. Geometric analysis of collaborative optimization. Structural and Multidisciplinary Optimization, 2008, 35: $301-313$.

[24] C. L. Gong, L. X. Gu. Design and implementation for missile overall design software system. Computer Simulation, 2012, 29(6): 71 - 74. (in Chinese)

[25] P. E. Gill, E. E. Wong, W. Murray, et al. User's guide for SNOPT version 7.4: software for large-scale nonlinear programming, 2015.

[26] R. S. Sellar, S. M. Batill, J. E. Renaud. Response surface based concurrent subspace optimization for multidisciplinary system design. Proc. of the 34th AIAA Aerospace Sciences and Meeting Exhibit, 1996.

[27] S. L. Padula, N. M. Alexandrov, L. L. Green. MDO test suite at NASA Langley research center. Proc. of the 6th AIAA, NASA, and ISSMO, Symposium on Multidisciplinary Analysis and Optimization, 1996.

[28] S. I. Yi, J. K. Shin, G. J. Park. Comparison of MDO methods with mathematical examples. Structural and Multidisciplinary Optimization, 2008, 35(5): $391-402$. 


\section{Biographies}

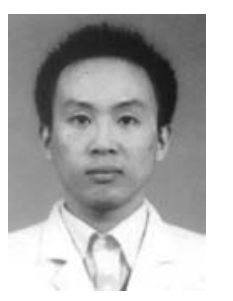

Hua Su was born in 1985. He is a Ph.D. and an assistant researcher in Northwestern Polytechnical University. His research interests are multidisciplinary design optimization and application, aircraft conceptual design.

E-mail: su@nwpu.edu.cn

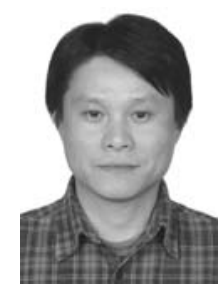

Chunlin Gong was born in 1980. He is a Ph.D. and an associate professor in Northwestern Polytechnical University. His research interests are aircraft conceptual design, multidisciplinary design optimization.

E-mail: leonwood@nwpu.edu.cn

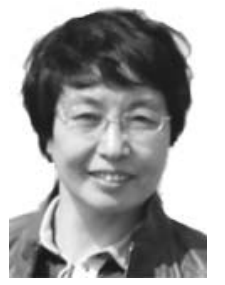

Liangxian Gu was born in 1957. She is a Ph.D. and a professor in Northwestern Polytechnical University. Her research interests are aircraft conceptual design, multidisciplinary design optimization.

E-mail: gulx@nwpu.edu.cn 